\title{
A fused mixed-methods approach to thematic analysis of personal networks: Two case studies of caregiver support networks
}

\author{
Reza Yousefi Nooraie ${ }^{1 *(1)}$, Bronwyn Thompson ${ }^{2}$, Chelsea D’Silva ${ }^{2}$, Ian Zenlea ${ }^{2,3,4}$, \\ Maryam Tabatabaee ${ }^{5}$ and Ardavan Mohammad Aghaei ${ }^{5}$ \\ ${ }^{1}$ Department of Public Health Sciences, School of Medicine \& Dentistry, University of Rochester, 265 Crittenden Blvd., CU \\ 420644, Rochester, New York 14642, USA, ${ }^{2}$ Institute for Better Health, Trillium Health Partners, Mississauga, Ontario, \\ Canada (e-mails: bronthomp88@gmail.com, chelsea.d'silva@thp.ca, Ian.Zenlea@thp.ca), ${ }^{3}$ Division of Children’s Health, \\ Trillium Health Partners, Mississauga, Ontario, Canada, ${ }^{4}$ Department of Pediatrics, University of Toronto, Toronto, \\ Ontario, Canada and ${ }^{5}$ Department of Psychiatry, Tehran University of Medical sciences, Tehran, Iran \\ (e-mails: marytabaee@gmail.com, ardavanaghaei@gmail.com) \\ *Corresponding author. Email: Reza_Yousefi-nooraie@URMC.Rochester.edu
}

Action Editor: Laura Koehly

\begin{abstract}
Thematic analysis of personal networks involves identifying regularities in network structure and content, and grouping networks into types/clusters, to allow for a holistic understanding of social complexities. We propose an inductive approach to network thematic analysis, applying the learnings from qualitative coding, fused mixed-methods analysis, and typology development. It involves framing (changing focus by magnifying, aggregating, and graphical configuration), pattern detection (identification of underlying dimensions, sorting, and clustering), labeling, and triangulating (confirmation and fine-tuning using quantitative and qualitative approaches); applied repeatedly and emergently. We describe this approach utilized in two cases of studying support networks of caregivers.
\end{abstract}

Keywords: network analysis; mixed methods; typology; ego-networks; caregiver networks

\section{Introduction}

Studying personal (egocentric) networks can provide important insights into the patterns and dynamics of social relations from the viewpoint of network actors. Similar to whole network (sociocentric) analysis, various structural measures have been developed to describe the composition of personal networks, while no single variable can sufficiently explain the complexity of social relations. This complexity results in the formation of patterns that can be better understood using a holistic approach. A pattern-centered approach to analyzing personal social networks (as opposed to a variable-centered approach) allows for simultaneous assessment of several variables and their complex relations (Antonucci et al., 2010). This process involves identifying patterns or themes that can explain similarities and differences across individual networks and then grouping networks into types based on similarities in structural and individual attributes.

A common pattern-centered approach to identify themes that classify and describe social networks is the development of network typologies. Through the process of developing a typology, social elements are classified into groups in which the elements share similar attributes. 
Developing typologies helps define complex concepts, explain underlying dimensions, and create categories for sorting, comparing, and testing hypotheses (Collier et al., 2012). Development of network typology reveals the underlying rules or forces that influence the formation of individual networks and makes the comparison between networks, societies, and time periods possible (Giannella \& Fischer, 2016). Comparison of network types across contexts, cultures, and relations will show common and unique social dynamics that drive observed patterns. Since the inception of network theories, scholars tried to classify networks into groups and types (Simmel, 1955), and as discussed below, over the years, used a host of quantitative, qualitative, and mixed-methods techniques to develop typologies of personal networks.

Wellman \& Potter (1999) drew attention to the under-explored notion of network typology.

More recently, in their seminal book on egocentric network analysis, Perry et al. (2018) pointed to the renewed need to return to studying network typologies. They argue that the recent shift from prioritizing overall patterns and big pictures to a more microscopic approach-using single measures of network structure-does not adequately capture the holistic patterns in social networks. This paper is a response to these calls; it is motivated by our experience with fused mixed-methods designs (Bazeley \& Kemp, 2012), and our previously published argument that network analysis is by nature a fused mixed-methods approach (Yousefi Nooraie et al., 2020). In this manuscript, we review the current approaches to developing personal network typologies and propose a novel, fused mixed-methods approach to developing network typologies. Using two case studies, we apply our proposed approach to thematic analysis of personal networks in two relevant yet unique contexts of studying personal networks of caregivers of children with obesity and caregivers to individuals with severe mental illness. Both cases illustrate caregivers' social support networks within two distinct cultural and health-related contexts characterized by different social structures and dynamics.

\section{Efforts on typifying personal networks}

Efforts to classify personal networks could be grouped into attribute-based and structural approaches (Bidart et al., 2018). Attribute-based typologies categorize networks based on the frequency of characteristics of network actors or relationships, such as the frequency of demographics (e.g. gender) or relationships (e.g. family-dominant networks or dominance of personal supportive ties). Structural categorization is about classifying personal networks based on their structural and network compositions, either overall (such as star-shaped or segregated, dense, or dispersed) or based on the structural characteristics of certain actors (such as existence of central or boundary spanner actors). As explained below, researchers often use a mix of attributebased and structural categorizations to develop typologies, using quantitative, qualitative, and mixed-methods approaches.

\subsection{Quantitative approaches}

Cluster analysis algorithms have been used to group personal networks into types (Antonucci et al., 2010), varying from simpler cluster analysis techniques to more complex such as latent class analysis (LCA) or random forest technique.

\subsubsection{Attribute-based approaches}

Some scholars used clustering techniques to develop typologies using attribute-based approaches. Agneessens et al. (2006) used LCA to classify 620 personal networks based on the type of support the respondents expected from different roles of their network actors. For example, they distinguished between networks in which the respondents expected companionship vs. when the respondents expected both companionship and emotional and/or instrumental support. 
Doeven-Eggens et al. (2008) used LCA to identify classes of 1,835 networks of university students based on the existence of various roles in their social networks (partner, parent, sibling, colleagues, friends, etc.) and identified three classes: primarily family, primarily peers, and mixed family/peers. Giannella \& Fischer (2016) used 43 variables (mostly attributes of the network actors, in addition to network density as the structural variable) from a large survey of personal networks. They applied a random forest technique to identify combinations of variables that best characterize particular subsets of personal networks of 1,050 individuals. After a multistep process of statistical optimization as well as relying on their "own substantive interpretation of the variable groupings" (p: 17), they ended up combining 21 variables into 7 composite dimensions (only including the attribute-based variables) that could describe the differences among personal networks, such as interaction with non-kin, proximity to kin, and overall involvement with and support from kin. Based on variables, they clustered personal networks into 11 types, including Career-and-Friends, Family-and-Community, Family-Only, Untethered, Energetic, Withdrawn, and Home-and-Church.

\subsubsection{Structural approaches}

Similar clustering techniques were also used to develop typologies using structural approaches. Lubbers et al. (2007) used k-means cluster analysis of standardized network structural measures (such as density, centralization, and number of cohesive subgraphs) of egocentric networks of 294 immigrants and explored solutions with different number of clusters. A five-cluster solution included scarce, dense family, multiple subgroups, two world's connected, and embedded networks. However, they did not clarify the process of labeling clusters. Brandes et al. $(2010,2011)$ studied the social networks of 504 immigrants and developed a typology based on the frequency of relationships within and across groups of network actors. They partitioned actors in each personal network into: in the origin country; from the origin country that also immigrated; in the host country; and others). They tried to identify structurally similar personal networks based on the size of partitions and their relationship, using cluster analysis, and interpreted the clusters in light of modes of acculturation in immigrants. They labeled clusters based on the overall patterns, such as strong separation, well-integrated, and assimilated. More recently, Vacca (2019) compared a subgroup-based method (community detection algorithm followed by typology detection using cluster analysis) vs. using cluster analysis on six predetermined structural measures. Applying 2 techniques on 6 datasets of 1,061 personal networks showed different results, revealing varying aspects of personal relations. He suggested guiding the typology methods by "theoretical considerations and substantive research applications" (p: 22).

In summary, scholars used various quantitative clustering algorithms to classify larger sets of personal networks based on structural- and attribute-based approaches. However, even in quantitative approaches, the statistical techniques were applied in subjective and inductive styles, which were not fully elaborated in most studies. Examples of this subjectivity include the process of developing the original list of variables, choosing the final variables from the initial list, potential development of composite variables and dimensions, the choice of clustering techniques, and labeling the resulting clusters and dimensions. We will explain how many of these processes could be informed by thematic analysis frameworks and techniques.

\subsection{Qualitative approaches}

Takahashi et al. (1997) qualitatively classified networks of 108 older people into several groups based on the existence of a dominant figure in the networks including family-dominant, frienddominant, and lone wolf. Wenger (1991) in a series of qualitative studies identified important factors to classify personal networks in older persons: the proximity of close kin, the proportion of family/fiends/neighbors, and the level of interaction between the individual and 
family/fiends/neighbors. Based on these factors, she classified personal support networks into: family-dependent (close relation with kin, usually based on shared household); locally integrated (close relation with family, friends, and neighbors, usually based on long-term residence and community involvement); locally self-contained (arm length relations, household lifestyle, and minimal community involvement); wider community-focused (active relationship with distant relatives, generally involved in community organizations, usually no local kin); and private restricted (minimal contacts). She developed an eight-item Practitioner Assessment of Network Type (PANT) questionnaire to assign individuals to the five network types, which has been crossvalidated in other contexts (Szabo et al., 2016). Morris et al. (2016) studied the personal networks of 30 individuals with long-term conditions. They applied a qualitative approach and developed network types by "combining the network composition and the narrative descriptions of the meanings that participants ascribed to relationships within their network" (p: 1048). They classified networks into friend-focused, family-focused, and health care professional-focused types.

Even though these studies explicitly identified network maps as qualitative data, none elaborated on the qualitative process of integrating network composition and narratives, or the process of coding, clustering, and identifying network types.

\subsection{Mixed-methods approaches}

There have been efforts to develop typology of personal networks by mixing qualitative and quantitative data. McCarty (2002) made one of the first efforts to develop a typology, by clustering the members of personal networks using a mixed-methods approach. He collected comprehensive information about personal networks from 45 individuals, each identified 60 network members, and then commented on the quality of relationship between all possible pairs. For each personal network, he conducted separate cluster analysis to group network actors, and calculated network structural measures such as density and various centrality measures. He then performed a "subjective exercise" "to determine why the members of the cluster would know each other" (pp: 13-14), by interpreting the structural- and attribute-based variables together. After coding 45 sets of clusters, he developed 12 major categories of individuals in personal networks: family; work together; network via other person; couples; school together; neighbors; social group; issue-oriented group; hobby group; religious affiliations; childhood; and housemates. He validated those clusters by member-checking with a small sample of respondents. Bidart et al. (2018) developed a mostly structural typology of personal networks. They applied a multistep and mixed-methods procedure on 287 personal networks from a longitudinal study. They developed a graphical presentation of networks by initially classifying networks by visual inspection ("the network looks like this, differs from that" [p: 5]) and then identifying the distinguishing factors qualitatively. During this process, they "trusted both [their] eyes and [their] knowledge of 'sociologically relevant' indicators" (p: 5). This process led to six prominent types of networks: regular dense (a densely connected network); centered dense (similar to previous with the addition of a central network actor); centered star (a centered network with loosely connected periphery); segmented (several relatively large and dense components and some isolated points); pearl collar (an elongated network with long chain of ties); and dispersed (fragmented into small groups and isolated individuals). The main distinguishing structural indicators that led to this clustering were density, betweenness centralization, segmentation, and diameter. Then, they performed a discriminant analysis to identify structural indicators that best classified personal networks into the typical networks at each of the six clusters.

The two mixed-methods studies used quantitative data obtained through clustering techniques to inform a holistic and subjective analysis of network patterns. However, they did not provide replicable details about the holistic analysis and the integration of quantitative and qualitative findings. 


\subsection{The need for a fused mixed-methods typology development}

Quantitative approaches have been mostly used to analyze larger networks, and many quantitative methods of classification of personal networks have been applied to personal networks that included 45 or more actors (Lubbers et al., 2007; McCarty, 2002). Clustering techniques such as LCA require large sample size and are especially suitable for identifying underlying dimensions and clusters in at least a few hundred personal networks (Gudicha et al., 2016). In smaller networks, statistical analyses are less informative. Additionally, in the majority of the quantitative clustering approaches, a list of attributes and structural measures were used. Even though most of these items were chosen based on theoretical and empirical relevance, the clustering technique itself was a stochastic algorithm that did not account for the underlying meanings and mechanisms. The qualitative approaches, on the other hand, have not been clear in terms of the actual process of analyzing and clustering personal networks. The few published mixed-methods studies have tried to overcome the limitations of quantitative clustering by applying complementary qualitative analysis. They recognized the need for a more intimate dialog between quantitative clustering and the subjective, inductive, and iterative process of meaning-making, as reflected by Bidart et al. (2018) referring to the process as "subjective exercise" requiring "trust[ing] both our eyes and our knowledge."

Consistent with other scholars, we believe that exploratory clustering algorithms, despite their quantitative appearance, involve several features of qualitative research such as the inductive and iterative nature of the analysis, the subjective/narrative process of choosing and refining variables, and the labeling of resultant clusters and dimensions (Jaworska \& Chupetlovska-Anastasova, 2009; Teddlie \& Tashakkori, 2009). Yousefi Nooraie et al. (2020) argued that social network analysis is a fitting example of fused mixed-methods analysis, in which the boundaries between quantitative and qualitative data and approaches are blurred. The mix of quantitative and qualitative approaches happen while choosing analytical techniques and network indicators (e.g. which centrality measure is relevant?), qualitative interpretation of data in graphs (e.g. what does the graph show about the social structures and dynamics?), and transformation of numerical output into meanings (e.g. labeling networks, regions, and actors based on their characteristics). In all these processes, meanings and narrative interpretations are closely interwoven with the quantitative measures. We extend this argument and propose that the pattern-centered analysis of social networks (e.g. typology development), even in its purely quantitative forms, is another fitting example of fused mixed-methods analysis.

We believe that pattern-centered analysis of networks can benefit from relevant traditions in other disciplines. The process of extracting meaning from the structural patterns in social networks in many occasions resembles various qualitative coding strategies (Saldaňa, 2016). Particularly, methods for the development of personal network typologies can benefit from other typology development frameworks. For example, the method proposed by Kluge (2000) for the development of empirically grounded typologies in qualitative research suggested a four-step approach of: developing dimensions; developing combination matrices for various attributes and assigning cases to various combination groups; selecting meaningful relationships and combinations; and describing resulting groups/types. We will discuss the proposed fused mixed-methods approach and will explain how some techniques resemble qualitative coding and thematic analysis, and transformation of codes into categories and concepts (Chowdhury, 2015).

\section{Proposed method}

Here we propose an inductive and exploratory thematic analysis of structural and attributebased patterns in personal networks. The proposed process involves techniques that we found potentially useful in the process of meaning-making of network patterns, which may be applied repeatedly, emergently, and selectively. The four main phases of the process involve framing, pattern detection, labeling, and triangulating (Figure 1): 


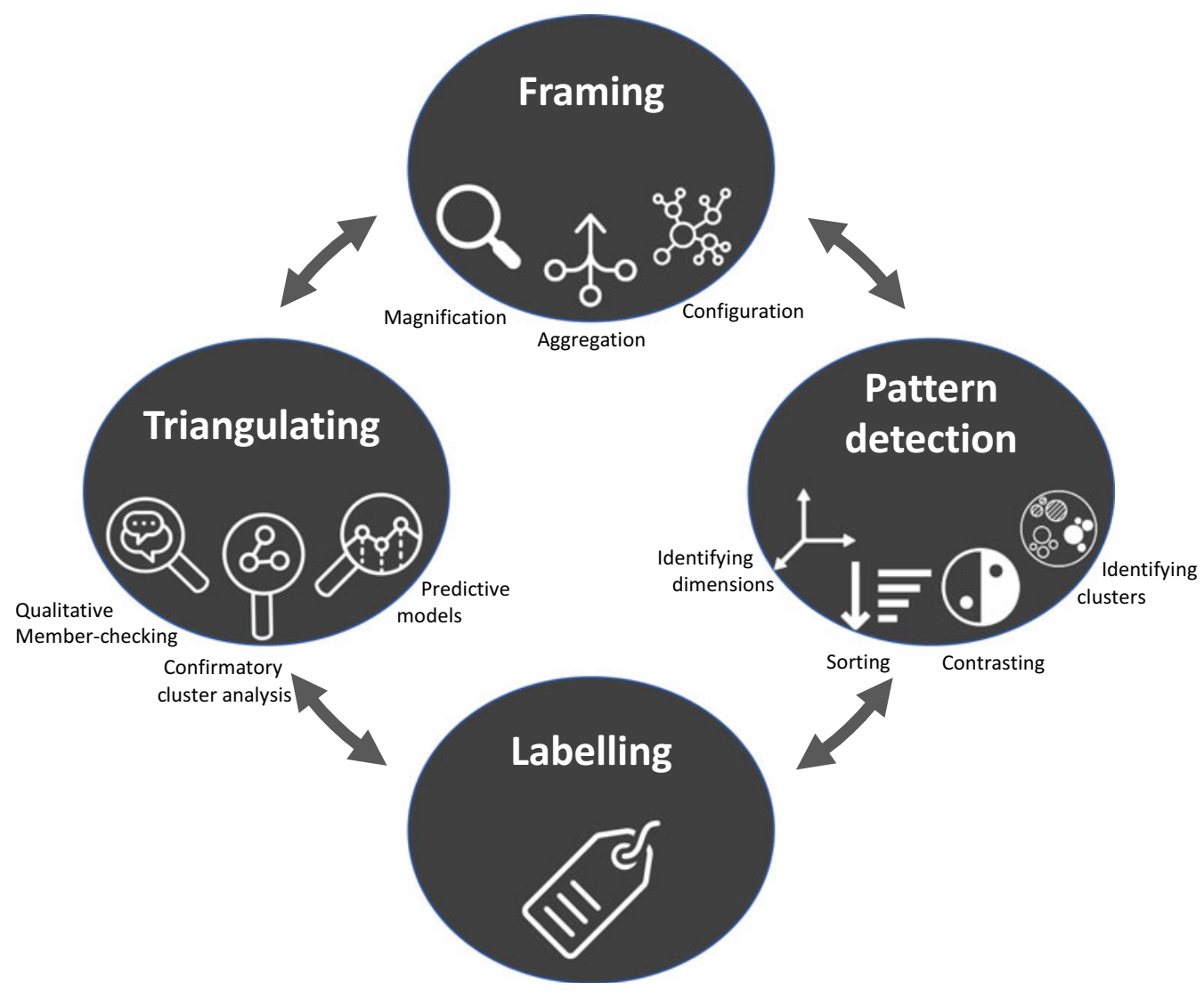

Figure 1. Schematic process of network thematic analysis. Icon credits: Dániel Aczél, Danil Polshin, Erik Arndt, Symbolon, Nun, Yu luck, and Meaghan Hendricks.

\subsection{Framing}

Network maps are visual presentations of complex social structures and dynamics. The process of framing is about directing the focus of attention to various aspects of this complexity. Framing may involve magnification (focusing on parts of the network), adjusting the graphical configuration (highlighting distances and distributions), and aggregating network actors/sections (adjusting the unit of analysis). This process resembles the process of identifying the unit of qualitative coding, for example, in line-by-line vs. passages-level coding, or the distinction between "splitting" and "lumping" (Bernard, 2013; Saldaňa, 2016). Some useful framing techniques include

- Magnification happens when the researcher adjusts the breadth and focus of the inspection. One can inspect the overall structure of the networks from the bird's eye view, studying the overall shape and composition (e.g. how centralized or clustered the network is), or may focus on certain individuals (e.g. how the spouse is located in the network in relation to ego and others) or on social roles (e.g. how health care providers are located in relation to ego and others).

- Aggregation happens when the network of individuals is collapsed based on social groups. The network measures may include within- and across-group densities (e.g. how frequently different groups communicate), and group centralities (which groups are more central). The aggregated network provides a more abstract and less detailed picture of social relations. 
- Graphical configuration is the process of changing the algorithm for the graphical presentation of the network maps. The process may involve moving back and forth between different force-directed layout algorithms (Venturini et al., 2019), concentric ego-network charts (Tubaro et al., 2016), and using various colors, shapes, and sizes to make the networks more informative about the structural attributes of the network (e.g. coloring different cohesive clusters, or sizing the nodes based on centralities), and individual and relational attributes (e.g. coloring the nodes based on gender, or changing the thickness of ties based on strength of relations).

\subsection{Pattern detection}

After framing the networks, the researcher tries to discover patterns. This process involves identification of dimensions against which the networks are distributed, and then two processes of sorting and contrasting. Identifying dimensions could be based on the overall shape of the network maps as well as their important and interesting features, which could be obtained from literature and/or the researcher's focus and interest. In prior studies, researchers have identified abstract dimensions as a step toward the development of network typologies (Giannella \& Fischer, 2016; Wellman \& Potter, 1999) and highlighted that network typologies represent a continuum of relationships with varying degrees (Morris et al., 2016). This process could be mapped onto several qualitative coding methods (Saldaňa, 2016):

- Exploring the overall shapes of network maps resembles holistic coding.

- Using known network characteristics (such as density, reciprocity, star-shaped, coreperiphery, or segregated networks, etc.) resembles provisional coding which relies on a predetermined list of characteristics.

- Identifying emergent features that were not in the initial lists resembles exploratory coding.

- Using nonnetwork features in the analysis, such as demographics or the position of interesting individuals and clusters (such as the position of health care providers in relation to others) resemble attribute coding and structural coding.

After identifying important dimensions (which itself is an iterative process), the researcher tries to sort the networks along dimensional axes. Sorting begins with the identification of extreme cases (two ends of the continuum) and typical cases (middle of the continuum). The other networks are then sorted in relation to the extreme and typical cases. The extreme cases will be placed on conceptual axes that help the sorting of the other networks that may display those patterns less prominently or have features of more than one axis. The sorting process could happen across several dimensions, and axes do not need to be placed perpendicularly. Contrasting is the process of comparing the identified dimensions across groups of individuals, based on some variables of interest. For example, we can compare the patterns in the support network of separated/divorced individuals with the individuals that live with a partner or compare among different levels of education, or various ethnicities.

After a few iterations of sorting and contrasting (and revising dimensions), the researchers should see how various personal networks are clustered together in the multidimensional conceptual space, with some clusters more distinct/visible than others. The process of identifying clusters resembles axial coding and theoretical coding, which involve holistic observation of the data (Saldaňa, 2016).

\subsection{Labeling}

At this phase, the detected patterns (either groups of personal networks or underlying dimensions) are labeled based on distinct and meaningful characteristics. This process of forming narrative 
profiles from numbers and patterns resembles "qualitization" (Teddlie \& Tashakkori, 2009). There are several methods for labeling groups of data based on analytical characteristics, which include the following:

- Average profiling: labeling based on average features. For example, a group of personal networks could be labeled as "dense networks" if they show high average density.

- Comparative profiling: labeling based on the comparison of groups. For example, the relative dominance of a social group could be used for labeling the type.

- Normative profiling: labeling based on comparing to a standard. For example, a network type could be labeled based on the extent by which it resembles a star network.

- Holistic profiling: labeling based on overall impression. For example, a network could be labeled as "strongly connected to health care providers" based on holistic judgment of researchers based on the multiplicity of health care providers and/or the strength of ties to them.

It is noteworthy that labels do not identify mutually exclusive aspects, and similar to thematic analysis, "could overlap and converge into various hierarchical themes and categories."

\subsection{Triangulating}

The thematic analysis of networks could be complemented by various quantitative and qualitative techniques. For example, dimensions identified through the coding process could be compared to a clustering algorithm, such as cluster analysis or LCA. As a member-checking process, qualitative interviews could also corroborate the patterns and their labeling from the viewpoint of the actors themselves. A group of raters can numerically score the networks based on the identified dimensions. The resulting dimensions could also be used as variables in statistical models to study their association with important individual characteristics (e.g. to assess if individuals with different network features vary in their health behavior, access to resources, quality of life, etc.).

\section{Two case studies}

We applied this method in two studies of social networks: Case 1. Caregivers of children with obesity in Canada; and Case 2. Caregivers of individuals with severe mental illnesses in Iran. Caregiving, especially to patients with chronic and severe illnesses, is a life-changing career, which imposes physical, mental, and financial burden on caregivers (Chang et al., 2010; Chappell \& Reid, 2002). Caregiving happens in the context of social networks, involving family members, friends, acquaintances, and sometimes paid care providers. Network members can reduce caregiver burden by providing instrumental and emotional support or contribute to caregiver distress through criticism, and stigmatization or by not providing support (Gresswell et al., 2018; Joyce et al., 2003). The distress can negatively impact the quality of the relationship between the caregiver and the person cared for as well as the broader social network around the caregiver. Studies in health care settings have shown that caregiver distress has been shown to increase morbidity and mortality in both in caregivers and patients (Schulz \& Beach, 1999). Despite the importance of the notion of caregiving and its inherently social nature, little is known about the structure of caregiver support networks. It is also noteworthy that most caregiving studies focus on "main caregivers" (often the spouse and/or parents) and miss the bigger picture and social nature of caregiving.

Caregivers' decisions and behaviors are influenced and scrutinized by their social network, and the structure of caregiver social networks may provide clues about the embeddedness of caregiving in social contexts (Roth, 2020). Existing network studies focus on variable-centered approaches to analyze caregivers' networks. Personal network size has been used frequently as a variable to surrogate network structure. Some studies showed that larger size and kin-dominant network 
were associated with better care quality (Fast et al., 2004). However, there is some evidence that size of the caregiver's support network may increase caregiving burden (Gresswell et al., 2018). The network size itself is affected by the duration of illness, and studies have shown the shrinkage of social networks in chronic diseases, or mental and cognitive illnesses (Anderson et al., 1984).

Very few studies used pattern-centered approaches to analyze caregiver networks. Keating \& Dosman (2009) used the Statistics Canada 2002 General Social Survey on Aging and Social Support data to develop a list of attribute-based indicators of caregivers' network. They used cluster analysis to group the networks into six clusters: children at home; spouse and children; lone spouse; older diverse; close kin and friends; and younger diverse. They highlighted the role of spouses and close kin as the main sources of bonding social capital, while friends could facilitate access to formal care and other resources. Bijnsdorp et al. (2019) used LCA to develop a typology of caregiving network of older adults and identified four types: partner network, mixed network, private network, and professional network. Neither of these studies assessed the structural aspects of social networks.

Little is known about the structural patterns of caregivers' embeddedness in social networks, and how the structure and composition of social networks may affect the caregiving process and caregivers' well-being.

\subsection{Case 1: Social Networks of Caregivers of children with obesity participating in a pediatric weight management program in Canada}

This mixed-methods study of social networks of parents of children with obesity participating in a pediatric weight management program aimed to investigate the structure and composition of support networks of parents, and their association with health behavior and perceived social influence. We conducted 22 semi-structured interviews with caregivers of children with obesity, who participated in a weight management program at Trillium Health Partners, Ontario, Canada. The demographic characteristics of study sample are provided in Table 1.

In an assisted process, the interviewees identified individuals who influenced their family's ideas of health and wellness and then distributed them on a network chart, depending on their social roles (family at home, family outside home, friends, colleagues and/or neighbors, health care providers, and others) and frequency of communication. The interviewees then drew lines between nodes who knew each other.

\subsubsection{Framing}

We used a force-directed algorithm to configure actors in personal networks, with different colors representing various social circles (adjusting the graphical configuration).

\subsubsection{Pattern detection}

We visually inspected the networks and identified extreme cases that showed prominent/distinct structural patterns (such as Figure 2(a) that resembles a high-density all-connected network, or Figure 2(b) that resembles a star). After a few rounds of sorting and clustering, we came to the conclusion that, perhaps instead of clustering networks, it would be more reasonable to identify dimensional axes representing specific features of networks, since many networks in the collection had features resembling more than one cluster.

\subsubsection{Labeling}

We defined the following dimensions representing network features:

- Cohesiveness: High score is represented by a network which is shaped by a dominant wellconnected circle of support, mostly including family and others who are also well connected to the family (example: Figure 2(a)). 
Table 1. Demographics of participants in Case 1 and Case 2

\begin{tabular}{|c|c|c|}
\hline & $\begin{array}{l}\text { Case 1: Caregivers to children participat- } \\
\text { ing in a weight management program }\end{array}$ & $\begin{array}{l}\text { Case 2: Caregivers to patients with severe } \\
\text { mental illness }(n=28)\end{array}$ \\
\hline Place & Ontario, Canada & Tehran, Iran \\
\hline Setting & Outpatient clinic & Inpatient psychiatry hospital \\
\hline Gender (female, \%) & $20(91 \%)$ & $16(60 \%)$ \\
\hline Post-secondary education & $18(81 \%)$ & $10(37 \%)$ \\
\hline Mean (SD) age & Not collected & $47(11)$ years; $\min =19, \max =63$ \\
\hline Relation to patient & Parent: $100 \%$ & $\begin{array}{l}\text { Mother: } 37 \% \\
\text { Spouse: } 26 \% \\
\text { Sibling: } 22 \% \\
\text { Child: } 11 \% \\
\text { Father: } 4 \%\end{array}$ \\
\hline Marital status & $\begin{array}{l}\text { Married or domestic partner: } 18(81 \%) \\
\text { Divorced or separated: } 3(14 \%) \\
\text { Widowed: } 1(5 \%)\end{array}$ & $\begin{array}{l}\text { Married: } 19(70 \%) \\
\text { Single: } 5(19 \%) \\
\text { Widowed: } 2(7 \%) \\
\text { Divorced: } 1(4 \%)\end{array}$ \\
\hline Characteristics of care recipients & $\begin{array}{l}\text { Children aged } 2-17 \text { years old with a body } \\
\text { mass index in the percentile range for } \\
\text { "obesity." }\end{array}$ & $\begin{array}{l}\text { Schizophrenia: } 13(50 \%) \\
\text { Bipolar mood disorder: } 9(35 \%) \\
\text { Schizo-affective: } 4(15 \%) \\
\text { Mean (SD) age: } 41(13) \text { years } \\
\text { Mean (SD) disease duration: } 20(10) \text { years } \\
\text { Female (\%): } 18(66 \%)\end{array}$ \\
\hline
\end{tabular}

(a)

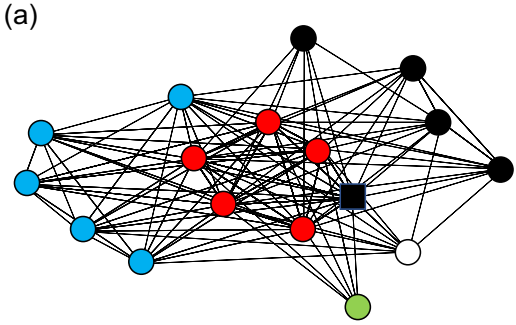

(c)

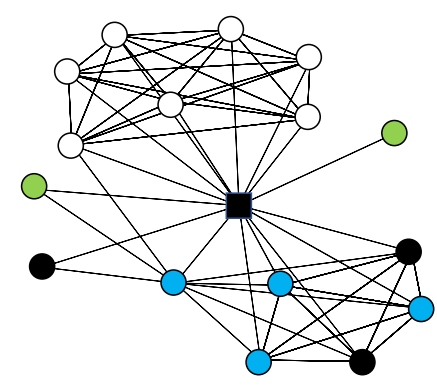

(b)

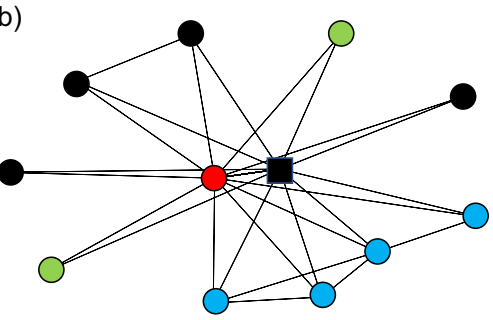

(d)

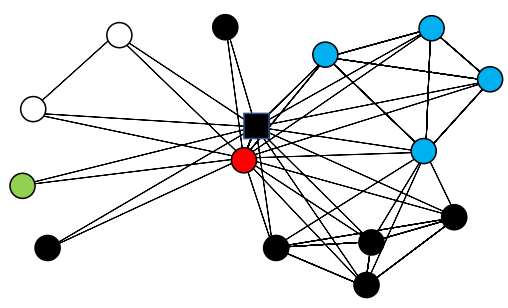

Figure 2. Examples of extreme networks along the four dimensions in Case 1. (a) High cohesiveness; (b) network resembling a star and existence of a core; (c) network with high clustering; (d) existence of a core. Black square: ego; red: family at home; blue: family outside home; black: friends; white: colleagues/neighbors; green: health care providers.

- Resemblance to star: Networks that score high have actors that are connected primarily to the ego or network core, with limited connections to one another (example: Figure 2(b)).

- Clustering: Networks that score high have distinct clusters with limited or no bridging ties (example: Figure 2(c)). 


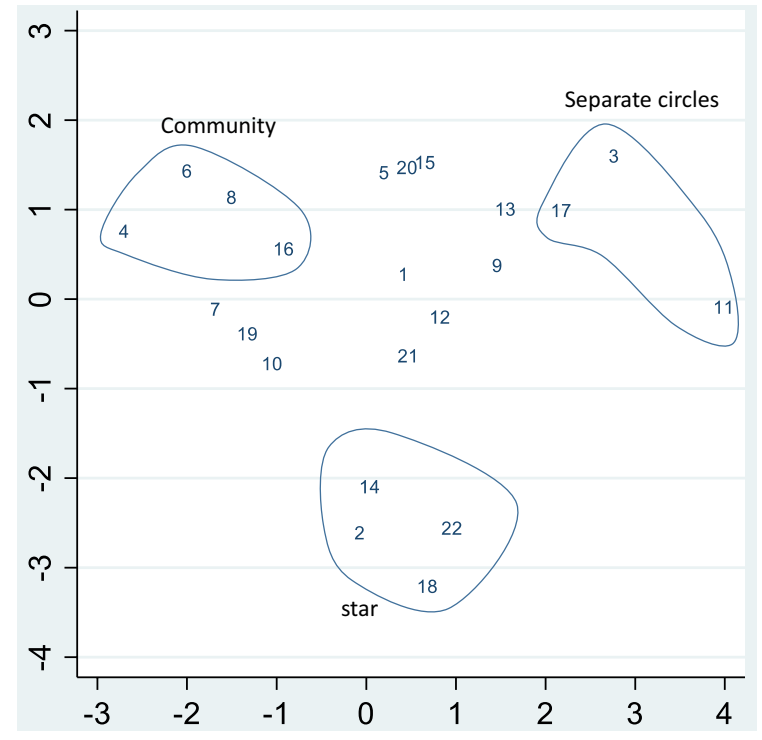

Figure 3. Multidimensional scaling of the scores to the four dimensions in Case 1. The numbers represent individual networks.

- Existence of a core: High score is represented by a network that has an actor or a few actors at the center who are highly connected to one another and the rest of the network. Usually, the core member was the spouse or partner. If you were to remove the ego, the network would remain largely intact due to the remaining core members (examples: Figure 2(a), (b) and (d)).

\subsubsection{Triangulation}

In order to see how much of the variability between the networks could be explained by these four dimensions, three authors visually inspected the graphical configurations of each personal network and separately scored (from 1 to 5) the extent to which each network represented the features of each dimensions, based on their subjective evaluation. We discussed and resolved disagreements, which resulted in some modification in defining dimensions. We used the fourdimensional scores of the 22 networks in a multidimensional scaling (MDS) process. MDS is a statistical technique that aims to provide a visual representation of the pattern of similarities among a set of objects by transforming score similarities to a proximity matrix and trying to fit the objects on a surface to best represent that matrix (Jaworska \& Chupetlovska-Anastasova, 2009; Teddlie \& Tashakkori, 2009). The MDS process distributed networks in a two-dimensional map, where the networks closer to each other had more similar features (Figure 3). We visually inspected the MDS map and labeled three distinguishable clusters as community, separate circles, and star. The "existence of a core" dimension did not result in a separate cluster, since networks that scored high on that axis were divided between community and star clusters. Networks with average scores on multiple axes located at the center of the map.

In summary, we followed the process of framing, pattern detection, labeling, and triangulating in an iterative and emergent manner. The analysis resulted in four dimensions and three distinct clusters of networks. Many networks represented features of multiple clusters. The dimensional scores identified in this analysis were used in a subsequent regression analysis to assess their association with the scores of social influence between ego and alters (under review). We found that alters were more likely to influence ego's healthy behavior in a network that resembled a star and less likely to influence ego in a network with a core member. We concluded that tightly knit relation to core member (e.g. spouse) potentially buffers the influence of other alters on ego, while people with star network selectively choose their diverse network members to address certain needs, while do not have access to the buffering network of close ties. 


\subsection{Case 2: Social Networks of Caregivers to individuals with severe mental illness in Iran}

This mixed-methods study aimed to explore the structure and dynamics of social support to caregivers of individuals with severe mental illness (schizophrenia, bipolar mood disorder, and schizo-affective disorder). Twenty-eight semi-structured interviews were carried out with primary caregivers of adults who were hospitalized for schizophrenia or bipolar disorder at an educational psychiatry hospital in Tehran, Iran. The demographic characteristics of study sample are provided in Table 1.

The caregivers were assisted in completing a network chart of individuals who supported them in providing care and the connections between these individuals. After an initial review of interviews and network charts, we realized that the personal support networks in this context were considerably smaller than Case 1 and were mostly dominated by the family members. We learned in qualitative interviews that the caregivers were particularly hesitant to form relationships outside of their family due to fear of being stigmatized by others, or reported unsuccessful experiences of prior connections, hence confined themselves with the immediate support groups.

\subsubsection{Framing}

Given the smaller size of the networks, we took a different approach to framing. Instead of exploring the individual-level networks, we aggregated the network actors into social roles: family at home, family outside home, friends, colleagues and/or neighbors, health care providers, and others. In the aggregated networks, each social role was represented by an arrow radiating from ego, with the thickness characterized the frequency and strength of connections at each slice.

\subsubsection{Pattern detection}

We used sorting and contrasting to detect patterns in aggregated networks. Similar to Case 1, we focused on identifying dimensions explaining the variability among aggregated maps, as networks represented several overlapping features, making them difficult to cluster.

\subsubsection{Labeling}

We identified and labeled the following dimensional axes:

- Multiplicity of social roles: a high score representing a network in which the ego was connected to various social roles (example: Figure 4(a) and (d))

- Dominance offamily: a high score representing a network in which the family is the only or the most important source of social support (examples: Figure 4(b), (c) and (d))

- Connectivity between social groups: a high score representing a network in which various groups all strongly connected (examples: Figure 4(b), (c) and (d))

- Overall strength of relations: a high score representing a network with strong relations between respondent (ego) and network actors (examples: Figure 4(c) and (d))

- Importance of health care providers: a high score representing a network in which health care provider role was important and also strongly connected to other roles (examples: Figure 4(c) and (d))

\subsubsection{Triangulation}

Similar to Case 1, three authors scored ( 1 to 5 ) the original individual-level networks along the five axes and discussed the disagreements. Figure 5 shows the MDS map of the agreed-upon scores, with labeling of various regions. We divided the map into main regions representing networks with weaker connections (left) or networks with a family-dominant feature (family as the only or main provider of the support). The weaker connections networks included diverse social roles (the 
(a)

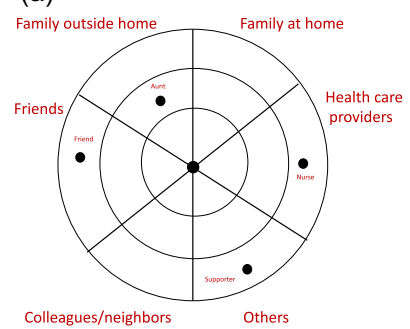

(c)

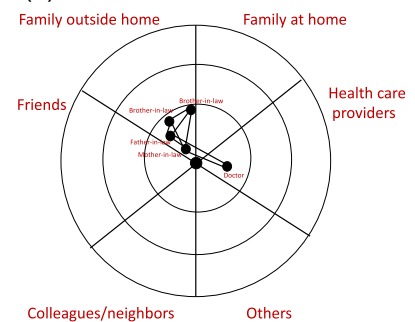

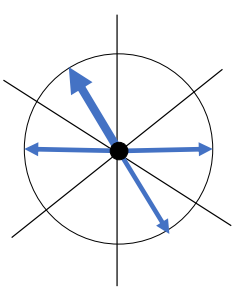

(b)

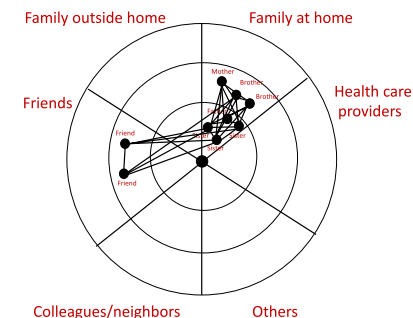

(d)

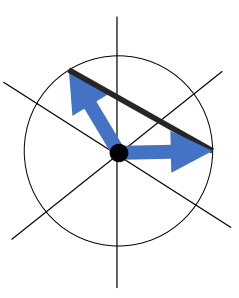

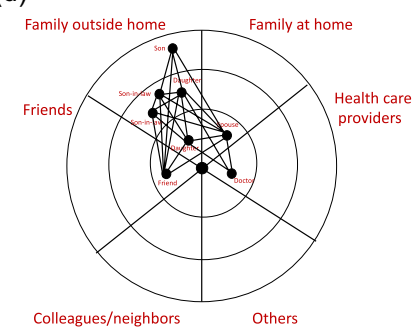
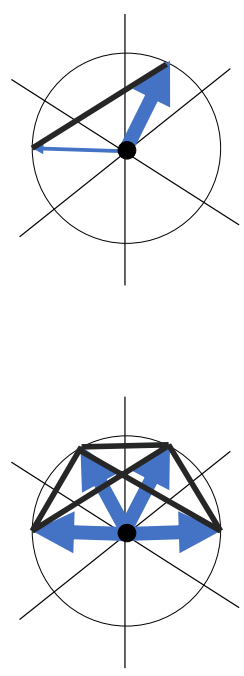

Figure 4. Examples of networks and their aggregated forms in Case 2. For each network, left map shows the actual presentation of network chart as developed by participant; right map shows the aggregated map based on social groups, with arrows representing the frequency and strength of connections between each social group and ego. (a) Multiplicity of social roles; (b) family dominance and connected social groups; (c) family dominance, connected social groups, strength of relations, importance of health care providers; (d) multiplicity of social roles, family dominance, connected social groups, strength of relations, and importance of health care providers.

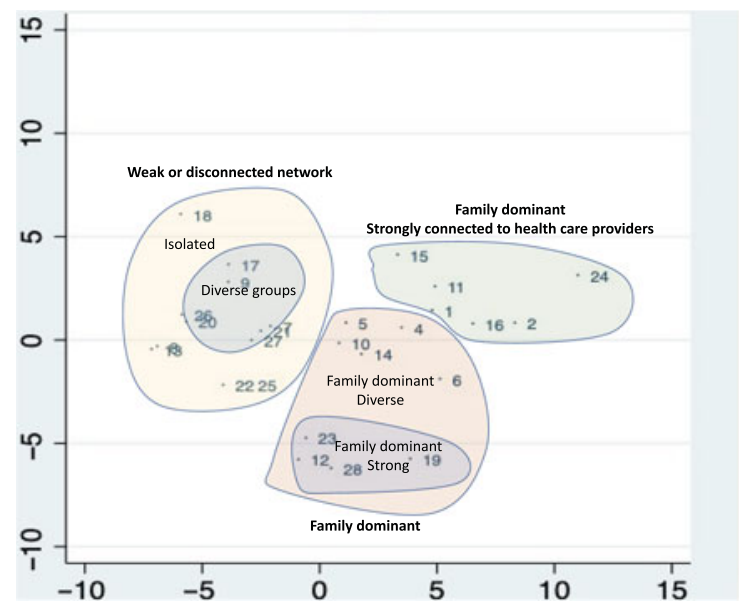

Figure 5. Multidimensional scaling of the scores to the five dimensions in Case 2. The numbers represent individual networks.

presence of family, friends, colleagues, etc.) or networks including very small number of actors (such as network \#18 in Figure 5 that only included one doctor). The family-dominant region was divided into three main sections: family-dominant networks with strong ties (bottom), familydominant networks with existence of diverse social roles (middle), and family-dominant networks with a strong connection to health care providers.

In summary, in this case, we used a different approach for framing the networks but followed the general procedure of pattern detection, labeling, and triangulating. Again, dimensional axes and network clusters provided overlapping but not redundant information about the variability of 
networks. We used the network dimensions in a subsequent qualitative analysis (in-progress), in which we developed the relationship of family dominance and importance of health care providers with themes related to stigma and caregiving burden. Our preliminary findings implied that the family dominance (especially when the caregiver is a mother) exacerbated the burden, despite our expectations of stronger support in tightly knit family networks, since the caregiver hesitated sharing the burden with other family members, while still being stigmatized within the family.

\section{Discussion}

We proposed a four-step approach for thematic analysis of egocentric networks, applying the learnings from qualitative coding, fused mixed-methods approaches, and typology development, while appreciating and recognizing the iterative, inductive, and mixed-methods nature of the process. The four-step approach involves framing (changing focus by magnifying, aggregating, and graphical configuration), pattern detection (identification of underlying dimensions, sorting, and clustering), labeling, and triangulating (confirmation and fine-tuning using quantitative and qualitative approaches). We demonstrated how these methods should be applied repeatedly and emergently. This approach provides a systematic framework to gain insight to personal network patterns.

The proposed method has common features with qualitative analysis of graphics. Scholars explored analyzing pictures and artistic artifacts as qualitative data (Saldaňa, 2016) and proposed semiotic analysis frameworks (to decipher meanings behind communicated signs) for qualitative coding of images (Gibson \& Brown, 2009; Grbich, 2013). While overlapping with qualitative analysis of images and artistic artifacts, the thematic analysis of personal networks has some special features that make it suitable fused mixed-methods analysis. A network map is a graphical presentation of a social world, rather than crafted artifact to communicate symbolic meanings, so the semiotic analysis is not an appropriate analytical framework. Alternatively, a network map presents several readily identifiable structural and quantitative features that could be analyzed through social network analysis methodologies and provide a rich collection of quantitative data. Network maps, which are essentially graphical representations of human relationship patterns, could accompany rich personal stories and perspectives obtained through qualitative inquiry (Dominguez \& Hollstein, 2014).

We applied this four-step approach in two culturally and substantively distinct contexts. Our case studies provide support for the usefulness of this structured approach. The personal networks in the case studies were different in terms of size and composition, which required two different framing techniques (a force-directed layout of personal networks in Case 1 and an aggregated presentation of networks in Case 2). In both cases, we followed a similar process of pattern detection (visual inspection, sorting, and contrasting) to identify underlying dimensions and network clusters. The resulting dimensions were overlapping but different in the two cases, reflecting the differences between the two contexts. The MDS analysis also showed differing distribution and clustering of the networks along the dimensions in two cases.

Generally, we found this approach to be particularly useful in studies with smaller sample sizes, which were not powered for advanced statistical techniques such as LCA, which generally requires a minimum of hundreds of personal networks. However, even with large samples, this approach could be used on subsets of data to reveal underlying dimensions, prominent clusters, and meaningful combination of variables to be used in statistical clustering.

The resulting structural dimensions of the two cases share some features, such as the existence of a core (mostly immediate family members), cohesiveness, and clustering. However, networks in the two cases showed distinct features that could be due to demographic and cultural differences, as well as the strength of disease-related stigma. Case 2 showed family dominance as a common feature in most networks, as the majority of personal networks were smaller and limited to family members. Limiting networks to family could be due to the stronger stigma of severe mental 
illness in traditional Iranian culture (Jacobsson \& Ghanean, 2013), which prevents caregivers from communicating with out-of-family individuals, mostly due to secrecy and withdrawal as coping mechanisms to reduce illness-related stigma (Link et al., 2004). However, another study of the caregivers in Case 1 showed that caregivers of children with obesity also experience stigma (Zenlea et al., 2017). On the other hand, caregivers of children with obesity reported more diverse social groups in their personal networks. These findings could be due to sociodemographic differences between two cases. Specifically, the sample of caregivers of individuals with mental illness (Case 2) included individuals with lower education mostly living a traditional lifestyle in a developing country, while the sample of caregivers of children with obesity (Case 1) were predominantly urban living, educated, and employed. Studies have shown that caregivers may use both bonding and bridging social capital to obtain support (Roth, 2020). Even though close family ties provide instrumental support, sometimes the tensions and the need for novel resources motivate caregivers to create new weaker ties (Knussen et al., 2005). This dynamic also depends on the stage of caregiving, as at early stages caregivers may disengage from peripheral networks and focus on the core, while over time, they may tap into nonredundant resources, to obtain novel support (Roth, 2020). In addition, the caregiving context in terms of life stage is very different between the cases. For Case 1, caregivers included parents (mostly mothers) providing care to their children, which fits well with traditional role expectations, whereas caregivers in Case 2 are more diverse, and many (such as siblings and spouses) may not find caregiving compatible with traditional expectations from their roles. The effect of social relations on the burden and quality of care is also a context and culture-specific issue, which is supported by some studies. Konerding et al. (2019), for example, found that being a spouse or at-home partner reduced caregiving burden in the UK and increased the burden in Greece.

The dominant role of health care providers in personal networks of Case 2 was another important distinction between the two cases; which could be interpreted as the result of more critical role of medications in controlling severe psychiatric symptoms, and stronger deference to doctors as authorities and experts in psychiatric care (Angell \& Bolden, 2015) and traditional Iranian culture (Sadati et al., 2016). The mentioned similarities and differences provide clues to network dynamics of caregiving, to be further explored in further studies.

The identified dimensions and network types could be used as classifying variables to assess common characteristics of individuals with similar network themes. For example, studies may assess varying needs and struggles of individuals with various network themes such as how the support dynamics and needs of caregivers in "community," "separate circles," or "star" network types differ in Case 1. Additionally, studying the association between personal and contextual variables with network themes facilitates the development of individualized and tailored interventions. Only a handful of studies assessed the effect of personalized network-building interventions to help vulnerable individuals expand and build more diverse support networks (Kennedy et al., 2015; Kennedy et al., 2016; van Asselt-Goverts et al., 2018). Network mapping in combination with motivational interviews and goal setting and reflection practices have been used to address social isolation and loneliness in older adults (O'Rourke et al., 2018). Personal network mapping could be used as a reflective activity to help caregivers optimize their support and informationsharing networks, activate dormant ties, or revisit unhelpful relations. For example, if studies show that people who are embedded in a dense family-dominant network (as observed in Case 2) may benefit from ties to outside individuals, then there might be benefit in developing targeted interventions to activate and strengthen external/weaker ties (such as colleagues, individuals dealing with similar issues, etc.). In addition, mapping influential network members provides opportunities to engage network members in the process of care and important decisions. Alternatively, providing a person's network map to health care professionals could potentially provide a better contextual picture of a person's network of care and support (Young et al., 2019) and influence and facilitate the achievement of "shared mind" (Epstein \& Street, 2011). This is an under-explored area of research, requiring more attention to the design and tailoring of such interventions. 


\section{Conclusion}

We proposed a four-step mixed-methods approach to thematic analysis of personal networks. It involves framing (choosing the focus of the attention, using techniques such as magnification, aggregation, and graphical configuration of networks) pattern detection (identification of conceptual dimensions, sorting and contrasting of personal networks, and identification of clusters), labeling (a qualitization process of transforming patterns to meaningful labels), and triangulating (which involves parallel approaches to further confirm and fine-tune the thematic analysis, including qualitative interviews, quantitative cluster analyses, or using themes/network types in predictive modeling). These steps can be followed in an iterative and circular fashion, which is consistent with fused mixed-methods approach to social networks (Yousefi Nooraie et al., 2020). Future methodological studies should focus on assessing the usefulness of various techniques of framing, patten detection, labeling, and triangulation, as well as usefulness of the identified themes and dimensional scores to predict clinical and behavioral outcomes. Further, a similar fused mixed-methods approach could be applied outside of network analysis to analyze other complex graphical presentations, such as radar charts (example in Forbes et al. (2017)), concept maps, and other graphical tools developed for organizing knowledge and learning (Eppler, 2006; Novak, 1990).

We believe that our fused mixed-methods approach to thematic analysis of personal networks expands the under-explored methodology of fused mixed-methods research. We also believe that a pattern-centered analysis of social networks provides a new perspective to study patterns and dynamics of personal networks.

Acknowledgments. We are grateful to Professor Ronald M. Epstein and Professor Supriya G. Mohile (University of Rochester) whose insightful feedbacks and suggestions were influential in formation of this article.

Author contributions. RYN conceptualized and developed the methodology, provided methodological oversight to data collection, led the analysis, and developed the manuscript drafts. IZ conceptualized and led Case 1. MT conceptualized and led Case2. BT, CD, IZ, MT, and AM conducted the data collection, participated in the analysis, and contributed in development of the manuscript. All authors reviewed and approved the final manuscript.

Ethical standards. Case 1 study was approved by Trillium Health Partners' Research Ethics Board and written consent was obtained from all participants. Case 2 study was approved by the Ethics Board of Tehran University of Medical Sciences, and written consent was obtained from all participants.

Conflict of interest. None.

\section{References}

Agneessens, F., Waege, H., \& Lievens, J. (2006). Diversity in social support by role relations: A typology. Social Networks, 28(4), 427-441. https://doi.org/10.1016/J.SOCNET.2005.10.001

Anderson, C., Hogarty, G., Bayer, T., \& Needleman, R. (1984). Expressed emotion and social networks of parents of schizophrenic patients. The British Journal of Psychiatry, 144, 247-255.

Angell, B., \& Bolden, G. (2015). Justifying medication decisions in mental health care: psychiatrists' accounts for treatment recommendations. Social Science \& Medicine, 138, 44-56.

Antonucci, T., Fiori, K., Birditt, K., \& Jackey, L. (2010). Convoys of social relations: integrating life-span and life-course perspectives. In R. Lerner, M. Lamb, \& A. Freund (Eds.), The handbook of life-span development. John Wiley \& Sons Inc.

Bazeley, P., \& Kemp, L. (2012). Mosaics, triangles, and DNA metaphors for integrated analysis in mixed methods research. Journal of Mixed Methods Research, 6, 55-72.

Bernard, H. R. (2013). Social research methods: Qualitative and quantitative approaches (2nd ed.). Sage.

Bidart, C., Degenne, A., \& Grossetti, M. (2018). Personal networks typologies: A structural approach. Social Networks, 54, $1-11$.

Bijnsdorp, F., Pasman, H., Francke, A., Evans, N., Peeters, C., \& van Groenou, M. (2019). Who provides care in the last year of life? A description of care networks of community-dwelling older adults in the Netherlands. BMC Palliative Care, 18,41 .

Brandes, U., Lerner, J., Lubbers, M., McCarty, C., Molina, J., \& Nagel, U. (2010). Recognizing modes of acculturation in personal networks of migrants. Procedia-Social and Behavioral Sciences, 4, 4-13. 
Brandes, U., Lerner, J., \& Nagel, U. (2011). Network ensemble clustering using latent roles. Advances in Data Analysis and Classification, 5(2), 81-94.

Chang, H.-Y., Chiou, C.-J., \& Chen, N.-S. (2010). Impact of mental health and caregiver burden on family caregivers' physical health. Archives of Gerontology and Geriatrics, 50(3), 267-271. https://doi.org/10.1016/j.archger.2009.04.006

Chappell, N., \& Reid, R. (2002). Burden and well-being among caregivers: examining the distinction. Gerontologist, 42, 772-780.

Chowdhury, M. (2015). Coding, sorting and sifting of qualitative data analysis: Debates and discussion. Quality \& Quantity, 49(3), 1135-1143.

Collier, D., LaPorte, J., \& Seawright, J. (2012). Putting typologies to work: Concept formation, measurement, and analytic rigor. Political Research Quarterly, 65(1), 217-232.

Doeven-Eggens, L., De Fruyt, F., Hendriks, A., Bosker, R., \& Van der Werf, M. (2008). Personality and personal network type. Personality and Individual Differences, 45, 689-693.

Dominguez, S., \& Hollstein, B. (Eds.). (2014). Mixed methods social networks research: Design and applications. Cambridge University Press.

Eppler, M. (2006). A comparison between concept maps, mind maps, conceptual diagrams, and visual metaphors as complementary tools for knowledge construction and sharing. Information Visualization, 5(3), 202-210.

Epstein, R. M., \& Street, R. L. (2011). Shared mind: Communication, decision making, and autonomy in serious illness. Annals of Family Medicine, 9(5), 454-461. https://doi.org/10.1370/afm.1301

Fast, J., Keating, N., Otfinowski, P., \& Derksen, L. (2004). Characteristics of family/friend care networks of frail seniors. Canadian Journal on Aging, 23, 5-19.

Forbes, G., Loudon, K., Treweek, S., Taylor, S., \& Eldridge, S. (2017). Understanding the applicability of results from primary care trials: lessons learned from applying PRECIS-2. Journal of Clinical Epidemiology, 90, 119-126.

Giannella, E., \& Fischer, C. (2016). An inductive typology of egocentric networks. Social Networks, 47, 15-23.

Gibson, W., \& Brown, A. (2009). Working with qualitative data. Sage.

Grbich, C. (2013). Qualitative data analysis: an introduction. Sage.

Gresswell, I., Lally, L., Adamis, D., \& McCarthy, G. M. (2018). Widening the net: exploring social determinants of burden of informal carers. Irish Journal of Psychological Medicine, 35(1 PG-43-51), 43-51. https://dx.doi.org/10.1017/ipm.2017.36

Gudicha, D., Tekle, F., \& Vermunt, J. (2016). Power and sample size computation for Wald tests in latent class models. Journal of Classification, 33, 30-51.

Jacobsson, L., \& Ghanean, H. (2013). Internalized stigma of mental illness in Sweden and Iran-A comparative study. Open Journal of Psychiatry, 3(4), Article ID: 36817.

Jaworska, N., \& Chupetlovska-Anastasova, A. (2009). A review of multidimensional scaling (MDS) and its utility in various psychological domains. Tutorials in Quantitative Methods for Psychology, 5, 1-10.

Joyce, J., Leese, M., Kuipers, E., Szmukler, G., Harris, T., \& Staples, E. (2003). Evaluating a model of caregiving for people with psychosis. Social Psychiatry and Psychiatric Epidemiology, 38(4), 189-195.

Keating, N., \& Dosman, D. (2009). Social capital and the care networks of frail seniors. Canadian Review of Sociology, 46, 301-318.

Kennedy, A., Vassilev, I., James, E., \& Rogers, A. (2015). Implementing a social network intervention designed to enhance and diversify support for people with long-term conditions. A qualitative study. Implementation Science, 11(1), 27.

Kennedy, D. P., Hunter, S. B., Osilla, K. C., Maksabedian, E., Golinelli, D., \& Tucker, J. S. (2016). A computer-assisted motivational social network intervention to reduce alcohol, drug and HIV risk behaviors among Housing First residents. Addiction Science \& Clinical Practice, 11, UNSP 4-UNSP 4.

Kluge, S. (2000). Empirically grounded construction of types and typologies in qualitative social research. Forum Qualitative Sozialforschung/Forum: Qualitative Social Research, 1(1), http://dx.doi.org/10.17169/fqs-1.1.1124.

Knussen, C., Tolson, D., Swan, I., Stott, D., \& Brogan, C. (2005). Stress proliferation incaregivers: The relationshipsbetween caregiving stressors and deterioration in family relationships. Psychology \& Health, 20(2), 207-221.

Konerding, U., Bowen, T., Forte, P., Karampli, E., Malmström, T., Pavi, E., ..., \& Gräßel, E. (2019). Do caregiver characteristics affect caregiver burden differently in different countries? American Journal of Alzheimer's Disease \& Other Dementias, 34(3), 148-152.

Link, B., Yang, L., Phelan, J., \& Collins, P. (2004). Measuring mental illness stigma. Schizophrenia Bulletin, 30(3), 511-541.

Lubbers, M., Molina, J. L., \& McCarty, C. (2007). Personal networks and ethnic identifications; The case of migrants in Spain. International Sociology, 22, 721-741.

McCarty, C. (2002). Structure in personal networks. Journal of Social Structure, 3, 1.

Morris, R., Kennedy, A., \& Sanders, C. (2016). Evolving 'self'-management: Exploring the role of social network typologies on individual long-term condition management. Health Expectations, 19(5), 1044-1061.

Novak, J. (1990). Concept mapping: A useful tool for science education. Journal of Research in Science Teaching, 27(10), 937-949.

O’Rourke, H., Collins, L., \& Sidani, S. (2018). Interventions to address social connectedness and loneliness for older adults: a scoping review. BMC Geriatrics, 18, 214. 
Perry, B., Pescosolido, B., \& Borgatti, S. (2018). Egocentric network analysis: Foundations, methods, and models. Cambridge University Press.

Roth, A. (2020). Informal caregiving and social capital: A social network perspective. Research on Aging, 42, 272-280.

Sadati, A., Tabei, S., Ebrahimzade, N., Zohri, M., Argasi, H., \& Lankarani, K. (2016). The paradigm model of distorted doctorpatient relationship in Southern Iran: a grounded theory study. Journal of Medical Ethics and History of Medicine, 9, 2.

Saldaňa, J. (2016). The coding manual for qualitative researchers (2nd ed.). Sage.

Schulz, R., \& Beach, S. (1999). Caregiving as a risk factor for mortality. JAMA, 282, 2215-2219.

Simmel, G. (1955). Conflict \& the web of group affiliations. Free Press.

Szabo, A., Stephens, C., Allen, J., \& Alpass, F. (2016). Construct validation of Wenger's support network typology. The Journals of Gerontology, 73(7), 1244-1249.

Takahashi, K., Tamura, J., \& Tokoro, M. (1997). Patterns of social relationships and PsychologicalWell-being among the Elderly. International Journal of Behavioural Development, 21, 417-430.

Teddlie, C., \& Tashakkori, A. (Eds.). (2009). Foundations of mixed methods research: Integrating quantitative and qualitative approaches in the social and behavioral sciences. Sage Publications Inc.

Tubaro, P., Ryan, L., \& D’angelo, A. (2016). The visual sociogram in qualitative and mixed-methods research. Sociological Research Online, 21(2), 1-18. https://doi.org/10.5153/sro.3864

Vacca, R. (2019). Structure in personal networks: Constructing and comparing typologies. Network Science (In press).

van Asselt-Goverts, A. E., Embregts, P. J. C. M., Hendriks, A. H. C., van Asselt-Goverts, A., Embregts, P. J. C. M., Hendriks, A. H. C., van Asselt-Goverts, A. E., Embregts, P. J. C. M., \& Hendriks, A. H. C. (2018). Evaluation of a social network intervention for people with mild to borderline intellectual disabilities. Journal of Applied Research in Intellectual Disabilities, 31(2), e229-e243. https://doi.org/10.1111/jar.12318

Venturini, T., Jacomy, M., \& Jensen, P. (2019). What do we see when we look at networks. An Introduction to visual network analysis and force-directed layouts. SSRN. http://dx.doi.org/10.2139/ssrn.3378438

Wellman, B., \& Potter, S. (1999). The elements of personal communities. In B. Wellman \& C. Boulder (Eds.), Networks in the global village; life in contemporary communities (pp. 49-82). Westview Press.

Wenger, G. (1991). A network typology: From theory to practice. Journal of Aging Studies, 5(2), 147-162.

Young, J., Poole, U., Mohamed, F., Jian, S., Williamson, M., Ross, J., ... Egan, T. (2019). Exploring the value of social network "care maps" in the provision of long-term conditions care. Chronic Illness, https://doi.org/10.1177/1742395319836463.

Yousefi Nooraie, R., Sale, J.E.M., Marin, A., \& Ross, L. E. (2020). Social network analysis: An example of fusion between quantitative and qualitative methods. Journal of Mixed Methods Research, 14(1), 110-124. https://doi.org/ $10.1177 / 1558689818804060$

Zenlea, I., Thompson, B., Fierheller, D., Green, J., Ulloa, C., Wills, A., \& Mansfield, E. (2017). Walking in the shoes of caregivers of children with obesity: supporting caregivers in paediatric weight management. Clinical Obesity, 7(5), 300-306.

Cite this article: Yousefi Nooraie R., Thompson B., D’Silva C., Zenlea I., Tabatabaee M. and Mohammad Aghaei A. (2021). A fused mixed-methods approach to thematic analysis of personal networks: Two case studies of caregiver support networks. Network Science 9, 236-253. https://doi.org/10.1017/nws.2021.4 